\title{
Clinical Features and Surgical Treatment of Schwannoma Affecting the Base of the Tongue: A Systematic Review
}

\author{
Jenna Lamendola Sitenga ${ }^{1}$ Gregory Alan Aird ${ }^{1}$ Austin Nguyen ${ }^{1} \quad$ Adam Vaudreuil $^{1}$ Christopher Huerter ${ }^{1}$ \\ ${ }^{1}$ Department of Clinical and Translational Science, Creighton \\ University School of Medicine Ringgold Standard Institution, Omaha, \\ Nebraska, United States \\ Address for correspondence Jenna Lamendola Sitenga, BSa, \\ Department of Clinical and Translational Science, Creighton University \\ School of Medicine, Medical School, 2500 California Plaza, Omaha, \\ Nebraska 68102, United States (e-mail: jls65509@creighton.edu).
}

Int Arch Otorhinolaryngol 2017;21:408-413.

\begin{abstract}
Introduction Schwannomas of the head and neck account for $25-40 \%$ of all cases, with presentation at the base of the tongue as the most frequent site for intraoral tumors.

Objectives Here, a systematic review was conducted to include 15 cases of patients with schwannoma of the base of the tongue.

Data Synthesis Most patients presented with a single, painless, well-encapsulated nodule at the base of the tongue. These nodules were slow-growing, with an average of 13.3 months from onset to presentation. Most cases were accompanied by airway obstruction, indicated by symptoms of dysphagia, dysarthria, snoring, and sleep apnea.

Keywords

- base of tongue

- schwannoma

- neurilemmoma

- peripheral nerve sheath tumor

- systematic review Overall, the histological studies were consistent with a benign schwannoma with a palisading Antoni A and Antoni B pattern without malignant changes in cell morphology. These tumors were treated via complete surgical excision, and all cases achieved full remission by final follow-up.

Conclusion Surgical removal is the primary mode of treatment with excellent postoperative prognosis and rare instances of recurrence. Given the rarity of this tumor, this review of available case studies serves to comprehensively describe clinical presentation and surgical treatment approaches to tongue base schwannoma.
\end{abstract}

\section{Introduction}

Schwannomas, or neurilemmomas, are benign neoplasms that arise from Schwann cells that surround the peripheral, cranial, or autonomic nerve sheaths. ${ }^{1}$ These slow-growing, benign, encapsulated tumors arise when proliferating Schwann cells form a tumor that encompasses the nerve sheath. ${ }^{1,2}$ Although the etiology is unknown, some causative factors such as spontaneous growth, external injury, chronic irritation, or exposure to radiation have been hypothesized. ${ }^{2}$ Typically these growths are solitary lesions with no genetic or gender predisposition, although they may rarely occur in multiples when associated with neurofibromatosis. ${ }^{3}$

received

August 11, 2016

accepted

December 21, 2016

published online

February 13, 2017
Approximately $25-48 \%$ of reported schwannomas occur in the head and neck region of patients between 20-50 years of age. ${ }^{2,4}$ However, only $1 \%$ originate in the intraoral cavity, mostly involving the tongue. ${ }^{4}$ Schwannomas of the tongue present as a painless mass with conventional histology demonstrating Antoni A patterns of nuclear palisades surrounding Verocay bodies with alternating Antoni B patterns. ${ }^{5}$ The presence of S-100 protein in immune-histochemical staining is a classic marker for diagnostic confirmation and magnetic resonance imaging (MRI) is the gold standard for preoperative imaging. ${ }^{6}$ As schwannomas are typically benign, well circumscribed, and minimally invasive tumors, complete surgical excision is the standard of care for ensuring no recurrence. ${ }^{7}$

Copyright $\odot 2017$ by Thieme Revinter

Publicações Ltda, Rio de Janeiro, Brazil
License terms

()ㅇ (1) $\Theta \circledast$ 
Intraoral schwannomas commonly produce symptoms of throat discomfort, dysphagia, dysarthria, snoring, and impaired breathing. Complete surgical excision leads to favorable outcomes with no recurrence. ${ }^{2,8}$ About 130 cases of tongue schwannoma have been reported in the English literature, and of those, only 19 cases have been documented at the base of the tongue. ${ }^{5}$ Surgical resection of schwannomas located at the base of the tongue are inherently difficult due to limited operative exposure amidst intricate neurovascular anatomy, which may cause significant morbidity when damaged, such as impaired speech, aspiration, dysarthria, and dysphagia. ${ }^{9}$ Due to the rarity of this lesion and the difficulties inherent in complete excision, surgical complications may occur, including airway management, nerve preservation, and anesthetic complications. ${ }^{1,10}$

The present article will review and comprehensively analyze relevant literature and patient data to more definitely explain the treatment options and optimal surgical approaches for base of tongue schwannomas.

\section{Review of Literature}

\section{Literature Search}

We systematically searched the National Library of Medicine's PubMed database up to June 2016. The following search terms were used: "tongue" and "lingual" combined with "schwannoma," "neurilemmoma," "nerve sheath tumor," and "Schwann cell tumor." We screened titles and abstracts for possible inclusion, and subsequently, retrieved the full text of potentially relevant articles for review. We chosen articles based on the following criteria: it must be written in English, report cases using primary human subjects, and discuss malignancies of the base of the tongue only, not of

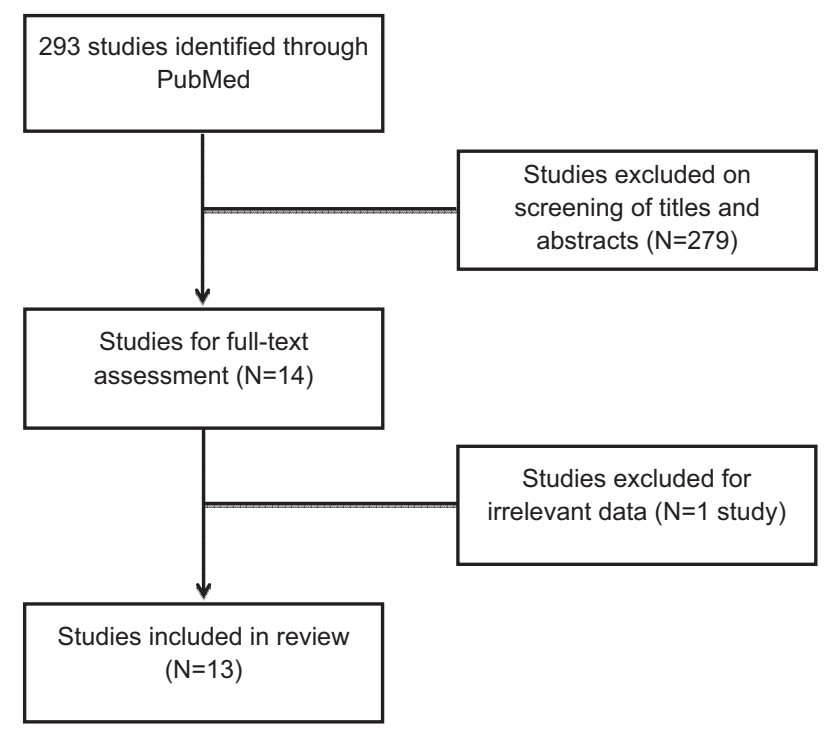

Fig. 1 Systematic search of PubMed returned 293 studies. After review of the titles, abstracts, and full-text, 13 studies were included in the present review. the palate, cheek, and other tongue regions. All other articles were effectively excluded.

\section{Results}

Initial search of PubMed returned 293 articles (see - Fig. 1). After screening the titles and abstracts for relevance, the full text of 14 articles were retrieved for review. Upon completion of full text review, we excluded one article because it was a literature review. Ultimately, 13 articles were included in this review, consisting of 15 cases total. All included articles were case reports $(N=11)$ or case series $(N=2)$. The highest number of cases was reported in India $(N=4)$, followed by the United States of America and Taiwan (both $N=3$ ). Summary of the study characteristics are reported in -Table 1.

\section{Clinical Presentation}

The mean patient age was 28.6 years (range $9-49$ years), and gender distribution nearly equal with 8 female patients (53.3\%) and 7 male patients (46.7\%). The clinical presentation of included cases is summarized in - Table 2 . All cases presented as a single nodule on the base of the tongue and all were symptomatic $(N=15,100 \%)$. Patients presented with either non-tender masses $(N=9,60 \%)$ or painful lesions $(N=6,40 \%)$ with reported discomfort at the back of the throat. Physical examination of the lesions revealed that the majority were smooth $(N=9,60 \%)$, non-ulcerated $(N=13,86.7 \%)$, round $(N=3,20 \%)$, well- defined and/or encapsulated $(N=13,86.7 \%)$ tumors. The most common symptomatic complaint was noticeable swelling $(N=13,86.7 \%)$ frequently causing

Table 1 Study characteristics and patient demographics

\begin{tabular}{|l|l|}
\hline Country & Studies (Cases) \\
\hline India & $4(4)$ \\
\hline Brazil & $1(1)$ \\
\hline USA & $3(3)$ \\
\hline Spain & $1(1)$ \\
\hline England & $1(1)$ \\
\hline Netherlands & $1(1)$ \\
\hline Sweden & $1(1)$ \\
\hline Taiwan & $1(3)$ \\
\hline Study Design & $11(11)$ \\
\hline Case report & $2(4)$ \\
\hline Case series & $\mathrm{N}($ range) \\
\hline Demographics & 15 \\
\hline Total patients & $28.6(9-49)$ \\
\hline Mean Age & 7 \\
\hline Gender & 8 \\
\hline Male & \\
\hline Female & \\
\hline
\end{tabular}


Table 2 Clinical presentation of tongue base schwannoma $(N=15)$

\begin{tabular}{|l|l|}
\hline Number of nodules & N (\%) \\
\hline Single & $15(100 \%)$ \\
\hline Well Defined & $13(86.7 \%)$ \\
\hline Non tender/painful & $9(60 \%)$ \\
\hline Pain/discomfort & $6(40 \%)$ \\
\hline Smooth & $9(60 \%)$ \\
\hline Submucosal & $4(26.7 \%)$ \\
\hline Firm & $4(26.7 \%)$ \\
\hline Symptoms & $13(86.7 \%)$ \\
\hline Swelling & $7(46.7 \%)$ \\
\hline Dysphagia & $5(33.3 \%)$ \\
\hline Dysarthria & $5(33.3 \%)$ \\
\hline Snoring & $3(20 \%)$ \\
\hline Compromised Airway & $2(13.3 \%)$ \\
\hline Impaired tongue mobility & $2(13.3 \%)$ \\
\hline Hemorrhage & $1(6.7 \%)$ \\
\hline Paresthesis & $1(6.7 \%)$ \\
\hline Otalgia & $13.3(r a n g e, 0.03-36)$ \\
\hline $\begin{array}{l}\text { Mean duration, months } \\
\text { (N = 12) }\end{array}$ & \\
\hline
\end{tabular}

dysphagia ( $N=7,46.7 \%)$, discomfort $(N=6,40 \%)$, dysarthria/change in voice $(N=5,33.3 \%)$, snoring $(N=5$, $33.3 \%)$, compromised airway and difficult nasal breathing $(N=3,20 \%)$, and impaired tongue mobility $(N=2,13.3 \%)$. Other symptoms during presentation included hemorrhage $(N=2,13.3 \%)$, cosmetic deformity $(N=1,6.7 \%)$, paresthesia $(N=1,6.7 \%)$, bilateral otalgia $(N=1,6.7 \%)$, and necrosis $(N=1,6.7 \%)$. A delayed visit to a medical professional was commonly seen, with a mean of 13.3 months (range 0.03-36 months) after the onset of symptoms to presentation.

\section{Workup}

Blood and urine chemistries were rarely reported in these case reports. The two cases in which blood and/or urine analyses were performed reported that all tests returned within normal limits. Biopsy was performed in 11 cases, the majority of which were done via excisional biopsy $(N=8 ; 72.7 \%)$ and the rest via fine needle aspiration ( $N=3 ; 27.3 \%$ ). All lesions were determined to be benign and none were graded as malignant tumors.

Of the cases that performed histological analyses, histological findings, shown in -Fig. 2 and -Fig. 3, of the biopsied tumors included spindle shaped or elongated cells $(N=13,100 \%)$ that were often palisading $(N=10$, $83.3 \%)$. Antoni A pattern was observed in 12 cases $(N=12$; $100 \%$ ) and Antoni B pattern was reported in 11 of those cases $(91.7 \%)$. Verocay bodies were found in 10 cases of those tumors that underwent biopsy examination
$(N=10 ; 100 \%)$. All of the cases were encapsulated, well-defined tumors $(N=15,100 \%)$ with four cases demonstrating dilated and congested vessels characteristic of schwannomas ( $N=4,50 \%$ present when checked for). Nine cases reported the use of immunostaining during evaluation. All cases stained positive for S100, while fewer cases stained positive for vimentin $(N=1,11 \%)$. No cases stained positive for actin, desmin, smooth muscle antigen, neuron specific enolase, or epithelial membrane antigen. The histological characteristics of tongue base schwannoma are outlined in - Table 3.

MRIs are the preferred imaging technique for diagnosing base of the tongue base schwannomas over other modalities due to higher spatial resolution and tissue contrast. ${ }^{3}$ Some form of imaging was performed in 13 cases, with MRI being the most common modality used $(N=9,69.2 \%)$. Magnetic resonance imagining, see - Fig. 4, revealed well-delineated, rounded lesions that were T2 hyperintense $(N=8,61.5 \%), \mathrm{T} 1$

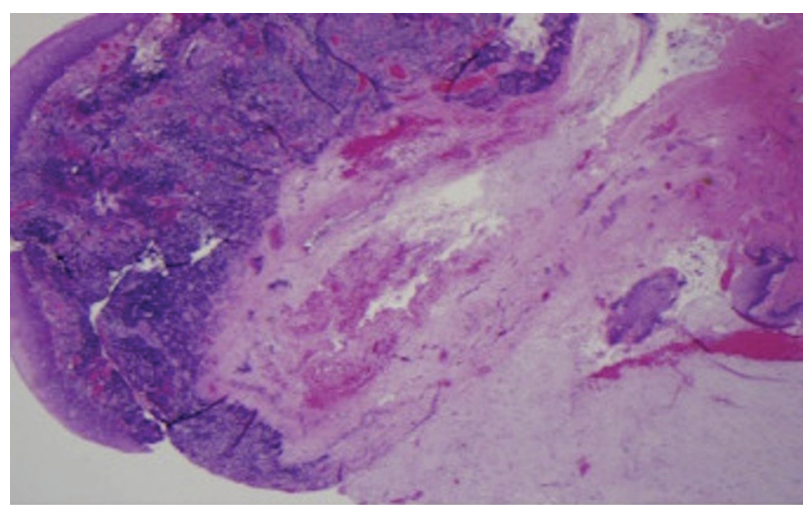

Fig. 2 Schwannoma of the tongue under low power, with underlying lymphocytic infiltrate and well-defined hypocellular lesion. Reproduced under CC-NC-ND from Badar et al (2016). ${ }^{15}$

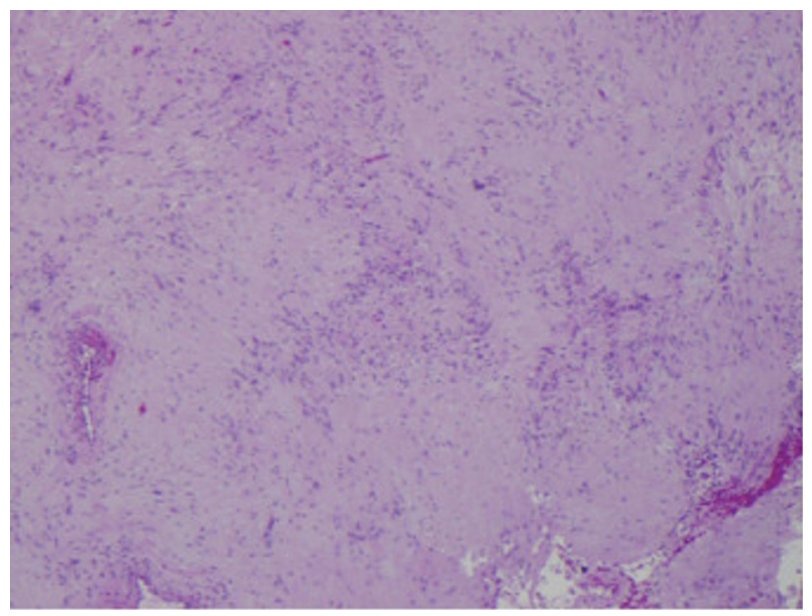

Fig. 3 Schwannoma of the tongue is composed of spindled cells with hypocellular and hypercellular regions in addition to focal nuclearpalisading areas (Verocay bodies). Reproduced under CC-NC-ND from Badar et al (2016). ${ }^{15}$ 
Table 3 Histological characteristics of tongue base schwannoma

\begin{tabular}{|l|l|}
\hline Histology (N varies by info reported) & N (\%) \\
\hline Spindle/elongated cells & $13 / 13(100 \%)$ \\
\hline Antoni A pattern or Verocay bodies & $12 / 12(100 \%)$ \\
\hline Palisading & $10 / 12(83.3 \%)$ \\
\hline Antoni B & $11 / 12(91.7 \%)$ \\
\hline Well delineated and/or encapsulated & $15 / 15(100 \%)$ \\
\hline Necrosis & $1 / 15(6.7 \%)$ \\
\hline Hemorrhagic & $2 / 15(13.3 \%)$ \\
\hline Dilated, congested vessels & $4 / 8(50 \%)$ \\
\hline Immunostaining (N $=15)$ & \\
\hline Positive S100; S100 diffuse staining & $15 / 15(100 \%)$ \\
\hline Radiology ( $N=13)$ & \\
\hline T2 Hyperintense & $8 / 13(61.5 \%)$ \\
\hline Hypointense T1 & $3 / 13(23 \%)$ \\
\hline Isointense to muscle & $3 / 13(23 \%)$ \\
\hline
\end{tabular}

hypointense $(N=3,23 \%)$, and isointense to muscle $(N=3,23 \%)$, all of which are enhancement patterns characteristic of schwannomas. Less common image findings included necrosis and blooming, indicating hemorrhage. All cases had well-defined margins, were well-encapsulated, and revealed no indication of invasion. Computed tomography was reported in 4 cases, and was primarily used to rule out metastases $(N=4)$.

\section{Outcome}

In a majority of the cases, preservation of nerve function was a primary concern during complete surgical resection. Despite a wide variety of surgical techniques utilized in the 15 cases, all postoperative recoveries were uneventful with no postsurgical complications such as loss of tongue function, sensation, or nerve damage. Of the papers that reported follow-up for their patients $(N=9)$, the mean follow-up period was 13 months (range 1.5 months- 60 months) with no evidence of recurrence in any of the cases.

The most common route used in resecting tongue base schwannomas was trans-orally $(N=7)$, which resulted in successful, short postoperative recovery times with no visible scarring, nerve damage, or loss of tongue function.

Another surgical approach was transcervical incision with blunt dissection $(N=1)$, resulting in a simple, quick procedure that protected surrounding nerves. Advantages to this approach included minimal morbidity, adequate exposure, no bony cuts, and minimal postoperative care.

One case utilized a suprahyoid pharyngotomy procedure $(N=1)$, during which a tracheotomy was first performed followed by an incision made at the hyoid bone, lacerating the platysma muscle, and enabling the removal of the suprahyoid muscles from the hyoid bone. This surgical technique led to a long postoperative period of 8 days and a more extensive and arduous recovery.

Other surgical approaches that were reported included mandibulotomy with lip splitting and mandibular swing $(N=1)$, which is unfavorable as it leaves aesthetically deforming scars around the mouth. Alternatively, the submandibular approach $(N=1)$ leaves a less noticeable scar below the mandible. Despite a resulting visible scar, the transhyoid approach $(N=1)$ allowed adequate resection and access to the base of the tongue with no morbidity and acceptable functional results. Excision via transoral laser microsurgery ( $N=2$ ) was used without any complication and the patient was discharged that same day postsurgery. Fiber optic bronchoscope (FOB)-guided awake nasotracheal intubation followed by general anesthesia was utilized in one case. The patient in this case was
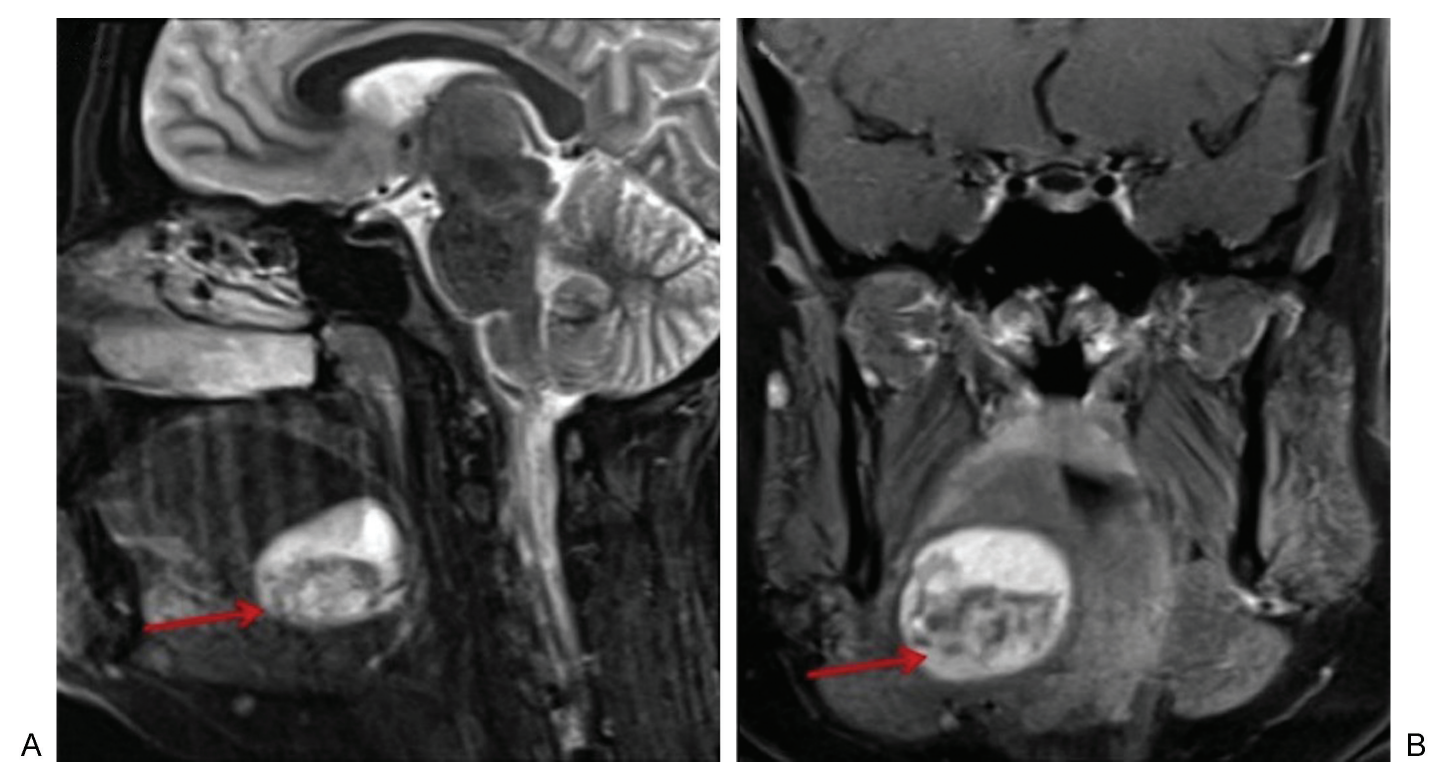

Fig. 4 Sagittal STIR MRI image of the head and neck (A) reveals a well-defined hyperintense mass (red arrow) within the base of the tongue. A coronal postcontrast fat-saturated image (B) shows heterogeneous enhancement of the lesion abutting the right submandibular gland. Reproduced under CC-NC-ND from Badar et al (2016). ${ }^{15}$ 
successfully intubated with no other complications following the procedure.

\section{Discussion}

The overall clinical presentation of tongue base schwannoma is fairly consistent, generally presenting as an encapsulated, well-defined mass at the back of the tongue. Oftentimes patients will delay seeking medical attention until the airway is compromised, presenting with symptoms of snoring, sleep apnea, difficulty breathing, dysphagia, and dysarthria. ${ }^{8-11}$ Generally, blood and urine chemistries are within normal limits. ${ }^{8}$ Biopsies and further histological analyses show characteristic histology of a schwannoma, revealing spindle shaped cells that are often palisading in a mixture of Antoni $A$ and Antoni B patterns. ${ }^{5,12}$ MRI investigation usually demonstrates a well-delineated, encapsulated lesion with enhancement patterns characteristic of schwannomas such as T2 hyperintensity, T1 hypointensity, and isointensity to muscle. ${ }^{11}$ All cases stain positively for $\mathrm{S} 100 .^{13}$ Complete surgical excision is the gold standard of treatment for schwannomas, leading to very low rates of recurrence. ${ }^{9,13}$

Surgical treatment of tongue base schwannomas is difficult due to limited operative exposure., ${ }^{9,10,14}$ Several surgical techniques are employed to remove schwannomas at the base of the tongue due to varying levels of surgical difficulty based on tumor size and location. ${ }^{14}$ Traditional surgical techniques for treatment of lesions at the base of the tongue include transoral excision, suprahyoid pharyngotomy, submandibular approach, and mandibulotomy with lip splitting. ${ }^{1-6,8-14}$ The transoral surgical approach is the most common and preferred method for small, well-encapsulated tumors, which do not cause any distortion of the airway. ${ }^{13}$ While transoral operations eliminate cosmetic disfigurement from scars, this technique is more likely to cause postoperative bleeding and swelling, especially in cases of larger, deeper lesions. ${ }^{14}$ of the traditional surgical methods used to treat tongue schwannomas, the transoral approach is recommended in favor of suprahyoid pharyngotomy and the submandibular approach, which should be reserved for schwannomas that are malignant or deemed too difficult to approach transorally. ${ }^{10,11}$ Although mandibulotomy with lip splitting is frequently used for treatment of larger schwannomas due to better exposure, this approach has a higher risk of damaging the intricate neurovasculature within this region and can cause significant morbidity. ${ }^{9}$

The utilization of laser treatment in tumor excision is an emerging alternative to traditional surgical methods. $\mathrm{ACO}_{2}$ laser surgery has high organ and nerve function preservation, minimal postoperative care and complications, and bypasses difficulties posed by excessive bleeding and obstruction of the lesion site. ${ }^{7}$ Laser surgery is very precise and readily seals any blood vessels, providing a dry field during dissection; however, higher cost and inadequate training of professionals limit this technique. ${ }^{7}$

Another surgical challenge inherent in tongue base schwannoma treatment is difficult intubation. Fiber optic broncho- scope-guided awake nasotracheal intubation followed by general anesthesia proved advantageous for schwannomas that profoundly obstructed the airway $(N=2)^{1,5}$ This technique was performed to preserve the airway anatomy and permit intact muscle tone to keep airway structures open, leading to easier visualization of the upper airway. ${ }^{1,5}$ Postoperative bleeding and swelling in the pharyngeal cavity and mouth floor is a common complication that can lead to dangerous obstruction of the airway after excision. ${ }^{1,5}$ Thus, nasal intubation and careful postoperative monitoring is recommended to maintain a viable airway even after a successful, complete excision. ${ }^{14}$ Proper anesthetic management for surgical excision of schwannoma of the tongue depends on the location, size, and surgical approach. ${ }^{1}$ Swellings located at the base of the tongue, though asymptomatic, may cause a distorted airway upon operative proceedings, causing life threatening airway obstruction after the induction of anesthesia. ${ }^{1}$

The methodology of this review was designed a priori with explicit procedures to reduce bias in article selection, appraisal, and data extraction and analysis. However, there exist limitations to the present review. Use of a single search database is one limiting factor. Additionally, the study is limited by the consistency of the literature in reporting data, even with thorough extraction of available data.

\section{Final Comments}

Tongue base schwannoma is a rare neoplasm with an excellent prognosis. The clinicopathological characteristics are consistent with that of benign peripheral nerve sheath tumors. Surgical removal is the primary mode of treatment with excellent postoperative prognosis and rare instances of recurrence. Preservation of lingual function and minimal postoperative complications, however still remain a challenge. Traditional surgical techniques, though routinely performed as the primary treatment, frequently result in poor potential outcomes such as swelling, scarring, bleeding, and longer recovery period. $\mathrm{CO}_{2}$ laser surgery is a new, upcoming treatment option that has been performed in several cases with excellent results. Investigation into the efficacy of this modality on primary lesions should be further explored as a minimally invasive alternative to traditional surgical treatment is highly desirable.

\section{Acknowledgements}

This research received no specific grant from any funding agency. This study did not involve human or animal subjects. The authors declare no conflicts of interest.

\section{References}

1 Batra UB, Usha G, Gogia AR. Anesthetic management of schwannoma of the base of the tongue. J Anaesthesiol Clin Pharmacol 2011;27(02):241-243

2 George NA, Wagh M, Balagopal PG, Gupta S, Sukumaran $\mathrm{R}$, Sebastian P. Schwannoma base tongue: Case report and review of literature. Gulf J Oncolog 2014;1(16):94-100 
3 Spandow O, Fagerlund M, Bergmark L, Boquist L. Clinical and histopathological features of a large parapharyngeal neurilemmoma located at the base of the tongue. ORL J Otorhinolaryngol Relat Spec 1999;61(01):25-30

4 Pereira LJ, Pereira PP, dos Santos JdeP, Reis Filho VF, Dominguete PR, Pereira AA. Lingual schwannoma involving the posterior lateral border of the tongue in a young individual: case report. J Clin Pediatr Dent 2008;33(01):59-62

5 Ballesteros F, Vilaseca I, Blanch JL, Gaspa A, Bernal-Sprekelsen $M$. Base of tongue neurilemmoma: excision by transoral laser microsurgery. Acta Otolaryngol 2007;127(09):1005-1007

6 Singh GB, Arora R, Garg S, Aggarwal K. Base of tongue schwannoma. ENT - Ear, Nose \& Throat Journal 2015:306-308

7 Mehrzad H, Persaud R, Papadimitriou N, Kaniyur S, Mochloulis G. Schwannoma of tongue base treated with transoral carbon dioxide laser. Lasers Med Sci 2006;21(04):235-237

8 Sharma S, Rai G. Schwannoma (neurilemmoma) on the base of the tongue: a rare clinical case. Am J Case Rep 2016;17:203-206
9 Ying YL, Zimmer LA, Myers EN. Base of tongue schwannoma: a case report. Laryngoscope 2006;116(07):1284-1287

10 de Bree R, Westerveld GJ, Smeele LE. Submandibular approach for excision of a large schwannoma in the base of the tongue. Eur Arch Otorhinolaryngol 2000;257(05):283-286

11 Sawhney R, Carron MA, Mathog RH. Tongue base schwannoma: report, review, and unique surgical approach. Am J Otolaryngol 2008;29(02):119-122

12 Bassichis BA, McClay JE. Pedunculated neurilemmoma of the tongue base. Otolaryngol Head Neck Surg 2004;130(05):639-641

13 Hsu YC, Hwang CF, Hsu RF, Kuo FY, Chien CY. Schwannoma (neurilemmoma) of the tongue. Acta Otolaryngol 2006;126(08): 861-865

14 Ying B, Zhu S, Qiao Y, et al. Surgical approaches for tongue base schwannoma. J Craniofac Surg 2013;24(01):e9-e11

15 Badar Z, Farooq Z, Zaccarini D, Ezhapilli SR. Tongue base schwannoma: differential diagnosis and imaging features with a case presentation. Radiol Case Rep 2016;11(04):336-340 